\title{
Behavioral Auditory Processing in Children and Young Adults with Autism Spectrum Disorder
}

\author{
Erin C. Schafer, $\mathrm{PhD}^{1}$ Lauren Mathews, $\mathrm{MS}^{1} \quad$ Kamakshi Gopal, $\mathrm{PhD}^{1} \quad$ Emilee Canale, BS \\ Avery Creech, BS ${ }^{1}$ Jacy Manning, BS ${ }^{1}$ Kara Kaiser, MS ${ }^{1}$ \\ ${ }^{1}$ Department of Audiology \& Speech-Language Pathology, University \\ of North Texas, Denton, Texas \\ J Am Acad Audiol 2020;31:680-689. \\ Address for correspondence Erin C. Schafer, PhD, \\ Erin.Schafer@unt.edu
}

\begin{abstract}
Keywords

- auditory processing

- autism spectrum disorder

- speech recognition

- binaural integration

- spatial stream segregation

Background Auditory-processing deficits are common in children and adults who are diagnosed with autism spectrum disorder (ASD). These deficits are evident across multiple domains as exhibited by the results from subjective questionnaires from parents, teachers, and individuals with ASD and from behavioral auditory-processing testing.

Purpose Few studies compare subjective and behavioral performance of adults and children diagnosed with ASD using commercially available tests of auditory processing. The primary goal of the present study is to compare the performance of adults and children with ASD to age-matched, neurotypical peers. The secondary goal is to examine the effect of age on auditory-processing performance in individuals with ASD relative to age-matched peers. Research Design A four-group, quasi-experimental design with repeated measures was used in this study.

Study Sample Forty-two adults and children were separated into four groups of participants: (1) 10 children with ASD ages 14 years or younger; (2) 10 age-matched, neurotypical children; (3) 11 adolescents and young adults with ASD ages 16 years and older; and (4) 11 age-matched, neurotypical adolescents or young adults.

Data Collection and Analysis Data from each participant were collected in one test session. Data were analyzed with analysis of variance (ANOVA), repeated measures ANOVA, or nonparametric analyses. Effect sizes were calculated to compare performance between those with ASD and those who were neurotypical within each age group.

Results Across all the questionnaires and the majority of the behavioral test measures, participants with ASD had significantly poorer ratings or auditory-processing performance than age-matched, neurotypical peers. Adults had more favorable performance than children on several of the test measures. Medium to large effect sizes corroborated the significant results.

Conclusion Overall, the questionnaires and behavioral tests used in this study were sensitive to detecting auditory-processing differences between individuals diagnosed with ASD and those who are considered neurotypical. On most test measures, children performed more poorly than adults. The findings in this study support that both children and adults with ASD exhibit auditory-processing difficulties. Appropriate school and work accommodations will be necessary to ensure appropriate access to speech in challenging environments.
\end{abstract}

received

December 2, 2019

accepted after revision

February 22, 2020

published online

December 14, 2020
(C) 2020. American Academy of

Audiology. All rights reserved.

Thieme Medical Publishers, Inc.,

333 Seventh Avenue, 18th Floor,

New York, NY 10001, USA
Dol https://doi.org/

10.1055/s-0040-1717138. ISSN 1050-0545. 
The Centers for Disease Control and Prevention report that approximately 1 in 59 eight-year-olds are identified with autism spectrum disorder (ASD), and approximately $80 \%$ of these children will receive special education services in the public schools. ${ }^{1}$ Given the large population of students diagnosed with ASD, appropriate special education services are costly and require specialized personnel to address the array of educational and sensory processing needs.

Individuals with ASD experience difficulties across various sensory domains; however, auditory filtering and processing is one of the most commonly reported issues. ${ }^{2-5}$ Auditory filtering is a critical skill in everyday environments because it defines a person's ability to hear, function, and complete tasks in the presence of background noise. Specific deficit areas reported by parents of children with ASD include difficulty attending to auditory stimuli, atypical auditory filtering, and dysfunction in noisy environments. ${ }^{2,5}$ Similarly, tests of auditory function reveal deficits in speech recognition in the presence of background noise, temporal processing, binaural integration (dichotic listening), and spatial stream segregation (i.e., use of spatial cues to improve performance). ${ }^{6-16}$ The magnitude of the speechin-noise deficits in various types of noise, expressed as signal-to-noise ratio (SNR), range from 2 to $8.3 \mathrm{~dB}$ relative to age-matched peers, which is notable given that $1 \mathrm{~dB}$ change is estimated to represent a $19 \%$ change in performance. ${ }^{6,12,14,17}$ These auditory deficits often coexist with multiple comorbid conditions common to children with ASD, including attention deficits, language disorders, and anxiety disorders.

Despite the documented auditory difficulties of children with ASD in previous investigations, there are limited studies comparing performance of individuals with ASD to neurotypical peers on commercially available tests of auditory-processing. An understanding of the expected auditory-processing deficits in children and adults with ASD will help determine an appropriate test battery as well as highlight areas of auditory-processing that may require intervention. When testing individuals with ASD, it is important to be cognizant of comorbid conditions that may impact performance. However, identifying performance deficits relative to peers, irrespective of specific causes, may help the audiologist and other related professionals to plan educational goals and objectives as well as potential interventions. The present study aims to (1) use commercially available tests and questionnaires to compare auditory-processing abilities in children and young adults with ASD to age-matched, neurotypical peers and (2) examine the effect of age on auditory-processing performance and subjective difficulties in individuals with and without ASD. Parent and participant questionnaires were used to assess auditory sensory processing issues and listening abilities in a classroom environment. The behavioral test measures assessed speech recognition in noise, auditory stream segregation of target and competing speakers, binaural integration with dichotic stimuli, and general hierarchical auditory processing.

\section{Methods}

\section{Participants}

Prior to the candidacy screening, parents of children $(\leq 14$ years) and adolescents or young adults ( $\geq 16$ years) agreed to participate by signing an institutional review board-approved consent form; children signed an assent form. Throughout the remainder of the manuscript, the word "adults" will be used to describe the group that included adolescents and young adults who were all attending college at the time of the study. The age cutoffs were chosen based on age criteria for one of the questionnaires. Study candidacy criteria included normal pure-tone air conduction thresholds $\leq 15 \mathrm{~dB}$ hearing level bilaterally from 250 to $8,000 \mathrm{~Hz}$ (audiometer: GSI-51; headphones: TDH-50), type A tympanograms (Maico MI34), and a nonverbal intelligence of $\geq 70$ on the Comprehensive Test of Nonverbal Intelligence Second Edition. ${ }^{18}$

Participants, ages 7 to 23 years, included 21 high-functioning individuals with a formal diagnosis of ASD ( - Table 1) (9 female; 11 male) and 21 aged-matched neurotypical peers (15 female; 6 male). Participants were assigned to child ( $\leq$ 14 years; $n=20$ ) or adult ( $\geq 16$ years; $n=22$ ) groups. According to parent report, participants with ASD were diagnosed by a licensed specialist in school psychology, pediatric neurologist, developmental pediatrician, or school diagnostician. All parents or participants reported a negative case history of otologic anomalies.

\section{Questionnaires and Test Measures}

The majority of participants ( $N=28 ; 7$ in each group) completed the Listening Inventory for Education (LIFE ${ }^{19}$ ), a self-report tool that identifies challenging classroom situations with a scale from 1 (always difficult) to 5 (always easy). One child with ASD was not able to reliably complete the LIFE, and despite multiple investigator requests, six participants did not return the LIFE. Parents of 10 participants in each child group, 14 years and younger, completed the auditory portion of the Child Sensory Profile $2,{ }^{20}$ a standardized tool to assess used sensory processing patterns at school, home, and in extracurricular activities. Ten participants in each group, ages 16 years and older, completed the auditory portion of the Adolescent/Adult Sensory Profile. ${ }^{21}$ One adult participant did not return this questionnaire. Raw scores were compared between the ASD and neurotypical groups.

The Bamford-Kowal-Bench Speech-in-Noise (BKB-SIN) ${ }^{22}$ sentence stimuli and four-talker babble on compact disc were presented in a sound-treated booth from a single loudspeaker at 0 degree azimuth. Results estimate the 50\% correct speech-in-noise threshold in dB SNR (i.e., SNR-50).

The Listening in Spatialized Noise - Sentences test (LiSN-S) 23,24 is administered via circumaural headphones (Sennheiser HD 215). In four LiSN-S conditions, the maskers are manipulated with respect to location (0 degrees vs. \pm 90 degrees azimuth) and vocal quality of the speaker (s) of the stories (same as, or different than, the speaker of the target sentences). Performance is measured as two speech 
Table 1 Demographic information for the participants with autism spectrum disorder (ASD)

\begin{tabular}{|c|c|c|c|c|}
\hline Group & Subject & Age $(y)$ & Sex & Other disabilities \\
\hline \multirow[t]{10}{*}{ Children } & 1 & 7.5 & M & $\mathrm{ADD} / \mathrm{ADHD}$ \\
\hline & 2 & 9.1 & $\mathrm{~F}$ & Language disorder \\
\hline & 3 & 9.5 & M & Did not complete form \\
\hline & 4 & 9.6 & $\mathrm{~F}$ & ADD/ADHD, APD \\
\hline & 5 & 10.2 & M & $\mathrm{N} / \mathrm{A}$ \\
\hline & 6 & 10.11 & M & APD \\
\hline & 7 & 12.0 & $\mathrm{~F}$ & Language disorder, specific language impairment \\
\hline & 8 & 13.11 & $\mathrm{~F}$ & ADD/ADHD \\
\hline & 9 & 14.3 & M & $\mathrm{N} / \mathrm{A}$ \\
\hline & 10 & 14.7 & M & ADD/ADHD, specific language impairment \\
\hline \multirow[t]{11}{*}{ Adolescents/Young adults } & 11 & 16.8 & M & ADD/ADHD, learning differences \\
\hline & 12 & 17.2 & $\mathrm{~F}$ & ADD/ADHD, language disorder, anxiety disorder \\
\hline & 13 & 20.6 & M & ADD/ADHD, APD, learning differences \\
\hline & 14 & 20.8 & M & $\mathrm{N} / \mathrm{A}$ \\
\hline & 15 & 21.3 & $\mathrm{~F}$ & ADD/ADHD, dyslexia \\
\hline & 16 & 21.5 & $\mathrm{~F}$ & $\mathrm{ADD} / \mathrm{ADHD}$ \\
\hline & 17 & 21.8 & $\mathrm{~F}$ & ADD/ADHD, anxiety disorder \\
\hline & 18 & 21.9 & M & $\mathrm{ADD} / \mathrm{ADHD}$ \\
\hline & 19 & 23.0 & $\mathrm{~F}$ & ADD/ADHD, depression, anxiety disorder \\
\hline & 20 & 23.5 & M & N/A \\
\hline & 21 & 23.5 & $\mathrm{~F}$ & ADD/ADHD, depression, anxiety disorder \\
\hline
\end{tabular}

Abbreviations: ADD, attention-deficit disorder; ADHD, attention-deficit hyperactivity disorder; APD, auditory processing disorder; $F$, female; $M$, male; N/A, not available.

reception threshold (SRT) measures and three "advantage" measures. These advantage measures represent the benefit in decibels gained when either talker, spatial, or both talker and spatial cues combined, are incorporated in the maskers.

The Randomized Dichotic Digits (RDDT) and Dichotic Words (DWT) tests, also administered with circumaural headphones (Sennheiser HD 215), assess free recall binaural integration abilities and provide digit/word scores for each ear as well as an ear advantage score (Audia Dichotic Software n.d.). The ear advantage score reflects asymmetry between dominant and nondominant ears irrespective of laterality. The DWT contains four lists of 25 word-pairs; the RDDT contains 54 presentations of one-, two-, or three-digit pairs.

General auditory-processing abilities of each participant were evaluated using the hierarchal Test of Auditory Processing Skills - Third Edition (TAPS- $3^{25}$ ). A repetition-response protocol was used to elicit a verbal response from participants following the clinician's lead (using live voice). Raw scores on these tests were used to compare performance of the ASD and neurotypical groups.

Prior to analysis, the data were separated into child ( $\leq$ 14 years; $n=20$ ) and adult groups ( $\geq 16$ years; $n=22$ ) to examine possible age effects across the questionnaires and test measures. Demographic information for the individuals with ASD is summarized in - Table 1. Most of the data were analyzed using a one-, two-, three-, or four-factor analysis of variance (ANOVA) or repeated measures ANOVA with independent variables including: group (ASD; neurotypical), age (child; adult), and condition when applicable (e.g., LiSN-S conditions). All data analyzed with an ANOVA had normal distributions according to the Shapiro-Wilk $W$ test or the D'Agostino Omnibus test to account for skewness or kurtosis of the data. The total number of factors is provided in the tables. Effect sizes (Cohen's $d$ ) were calculated ${ }^{26}$ between the ASD and neurotypical groups within each age group and are shown in the figures, tables, or provided in the text.

The RDDT and DWT data and three subtests on the TAPS did not have normal distributions according to Shapiro-Wilk $W$ test or the D'Agostino Omnibus tests. As a result, these data were analyzed with the Kruskal-Wallis test. This analysis does not calculate any interaction effects. Effect sizes for nonparametric data were calculated. ${ }^{27}$

\section{Results}

\section{Questionnaires}

Average performance on the LIFE $(N=28)$ and Sensory Profile $(N=40)$ questionnaires across the groups is illustrated in - Fig. 1. As shown in - Table 2, analyses of the average questionnaire ratings revealed significantly poorer auditory sensory processing and classroom listening abilities in 


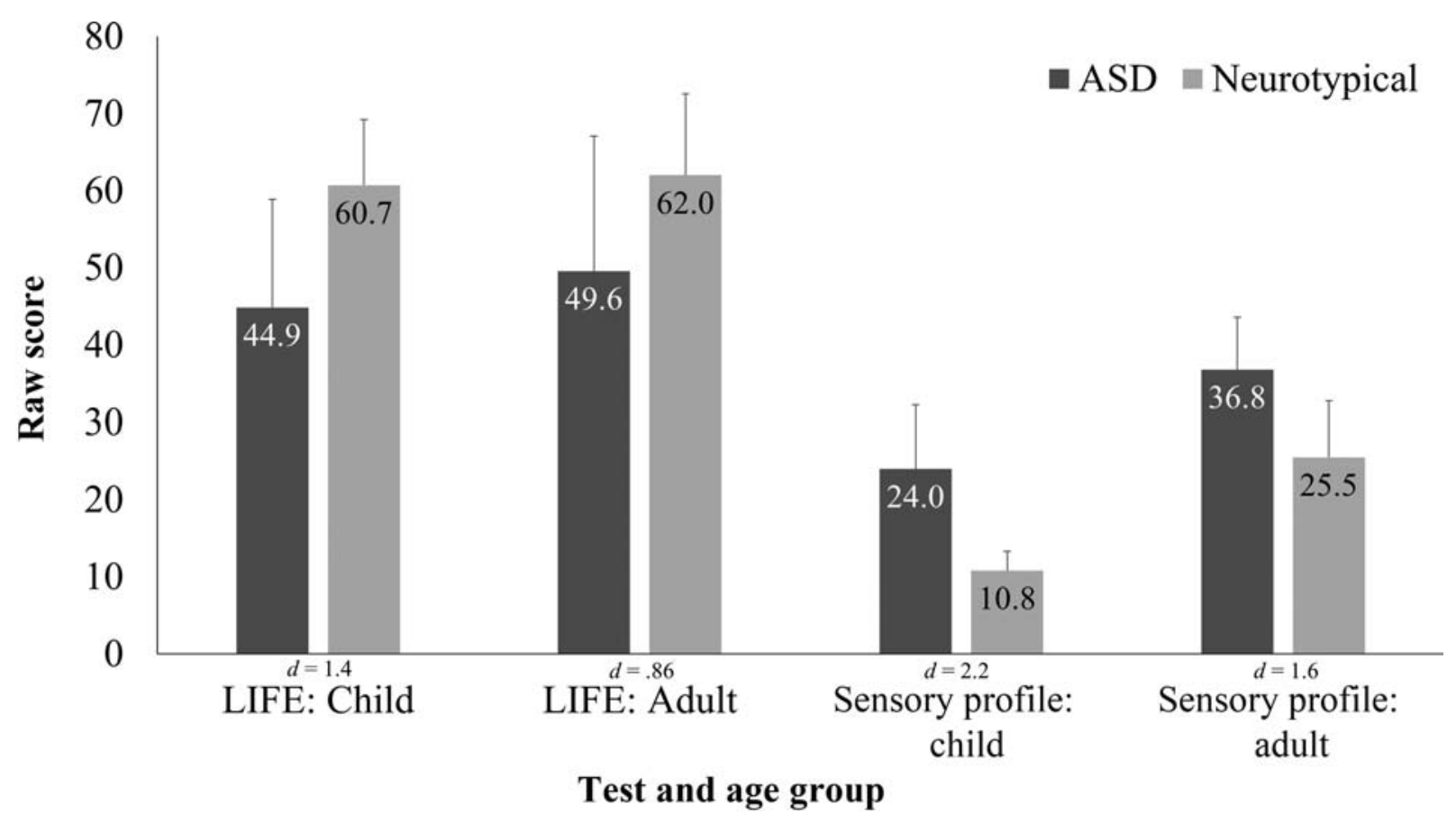

Fig. 1 Average raw scores on the Listening Inventory for Education (LIFE) questionnaire and auditory section of the Sensory Profile questionnaire. Effect sizes between groups are shown above the test and age group axis labels. Higher ratings on the LIFE indicate less listening challenges while higher ratings on the Sensory Profile indicate more frequent auditory sensory issues. Vertical bars represent one standard deviation. ASD, autism spectrum disorder.

individuals with ASD relative to the controls. Large effect sizes were observed for all group comparisons (-Fig. 1).

\section{Behavioral Measures}

Average speech-in-noise thresholds on the BKB-SIN data are provided in - Fig. $2(N=42)$. Analyses of these data (-Table 2) suggested significantly better performance for the adult groups as well as the neurotypical groups when compared with the child groups and groups with ASD, respectively. The within-age-group effect size was very large for the children and moderate for the adults ( - Fig. 2).

The average results of the LiSN-S test are shown in - Fig. 3 $(N=42)$. Analyses of the SRT conditions ( - Table 2 ) showed that, on average, the adult groups had lower (better) thresholds in noise than the child groups, the neurotypical groups had better thresholds than the groups with ASD, and the high-cue SRT was better than the low-cue SRT. Within each SRT condition and age group, effect sizes were large or very large (-Fig. 3). In the three advantage conditions (spatial, talker, and total), again, the adult groups showed greater advantages than the child groups, and the neurotypical groups showed greater advantages than the groups with ASD. In addition, there was a significant main effect of advantage condition with the total condition yielding the largest advantage followed by the spatial condition and talker condition. Effect sizes for the advantage conditions were large for children, but small to medium for the adults (-Fig. $\mathbf{3}$ ).

The results of the analyses on the RDDT and DWT $(N=42)$ are provided in $\mathbf{- T a b l e ~} \mathbf{3}$, and $\mathbf{- F i g .} \mathbf{4}$ illustrates the average results on the DWT. According to the nonparametric analyses of the RDDT, there was no significant effect of age or ear (i.e., dominant vs. nondominant), but the neurotypical group had significantly better digit recognition than the participants with ASD for all three-digit pairs. When averaging scores for the 1-pair, 2-pair, and 3-pair digits, effect sizes were large between the child neurotypical and ASD group (0.93-1.0) and between adult neurotypical and ASD group (0.91-1.1) for both the dominant and nondominant ears. When examining dominant and nondominant ear difference (i.e., ear advantage), the group with ASD had significantly larger difference scores between ears when compared with the neurotypical group for 2- and 3-pair digits as well as medium to large effect sizes between groups (child groups $=0.64$; adult groups $=0.89$ ). On the DWT, adults had higher scores than children (-Fig. 4), and the neurotypical group had higher scores than those with ASD with a large effect size for the children and medium effect size for the adults. There was no significant difference in ear advantage across the ages; however, there was a significant difference between the ASD and neurotypical groups, with the ASD group showing larger ear differences ( - Table 3 ).

The average data from the TAPS- 3 is provided in $-\mathbf{F i g . 5}$, and the results of the analyses are summarized in - Table 4 $(N=40)$. One adult with ASD was unable to complete the TAPS due to attention issues. On average, adults had significantly higher raw scores than the children on the word discrimination, phonological segmentation and blending, word memory, sentence memory, auditory comprehension, and auditory reasoning subtests. The neurotypical group had significantly higher raw scores on the phonological blending, number memory reversed, word memory, sentence memory, and auditory reasoning subtests. No interaction effects were 
Table 2 Summary of statistical analyses on questionnaires and behavioral test measures

\begin{tabular}{|c|c|c|c|c|c|}
\hline Test & Effects and factors & df & F-statistic & $p$ & Interpretation and post hoc results \\
\hline \multirow{3}{*}{$\begin{array}{l}\text { Listening Inven- } \\
\text { tory for Educa- } \\
\text { tion (LIFE) }\end{array}$} & -Main: Age & 1,27 & 0.37 & 0.55 & No effect \\
\hline & -Main: Group & 1,27 & 8.1 & $0.01^{\mathrm{a}}$ & Neurotypical better than ASD \\
\hline & -Interaction & 1,27 & 0.12 & 0.73 & No interaction \\
\hline $\begin{array}{l}\text { Sensory Profile } \\
\text { child }\end{array}$ & -Main: Group & 1,20 & 23.6 & $<0.001^{\mathrm{a}}$ & Neurotypical less difficulty than ASD \\
\hline $\begin{array}{l}\text { Sensory Profile } \\
\text { adult }\end{array}$ & -Main: Group & 1,20 & 12.9 & $0.01^{\mathrm{a}}$ & Neurotypical less difficulty than ASD \\
\hline \multirow[t]{3}{*}{ BKB-SIN } & -Main: Age & 1,42 & 14.2 & $0.01^{\mathrm{a}}$ & Adults better than children \\
\hline & -Main: Group & 1,42 & 7.2 & $0.01^{\mathrm{a}}$ & Neurotypical better than ASD \\
\hline & -Interaction & 1,42 & 2.3 & 0.14 & No interaction \\
\hline \multirow{16}{*}{$\begin{array}{l}\text { Listening in Spa- } \\
\text { tialized Noise } \\
\text { Sentences Test } \\
\text { (LiSN-S) }\end{array}$} & SRT Cond: & & & & \\
\hline & -Main: Age & 1,84 & 13.3 & $0.01^{\mathrm{a}}$ & Adults better than children \\
\hline & -Main: Group & 1,84 & 4.8 & $0.03^{\mathrm{a}}$ & Neurotypical better than ASD \\
\hline & -Main: Cond & 1,84 & 1098.0 & $0.01^{\mathrm{a}}$ & High- better than low-cue SRT \\
\hline & -Interaction: & & & & \\
\hline & Age/Group & 1,84 & 1.2 & 2.7 & No interaction \\
\hline & Group/Cond & 1,84 & 0.29 & 0.59 & No interaction \\
\hline & Age/Cond & 1,84 & 0.47 & $0.04^{\mathrm{a}}$ & All different except low-cue \\
\hline & Adv Cond: & & & & \\
\hline & -Main: Age & 1,126 & 11.9 & $0.01^{\mathrm{a}}$ & Adults better than children \\
\hline & -Main: Group & 1,126 & 4.3 & $0.05^{\mathrm{a}}$ & Neurotypical better than ASD \\
\hline & -Main: Cond & 2,126 & 148.6 & $0.01^{\mathrm{a}}$ & Total $>$ Spatial $>$ Talker \\
\hline & -Interaction: & & & & \\
\hline & Age/Group & 1,126 & 0.99 & 0.33 & No interaction \\
\hline & Group/Cond & 2,126 & 0.17 & 0.85 & No interaction \\
\hline & Age/Cond & 2,126 & 2.4 & 0.09 & No interaction \\
\hline
\end{tabular}

Abbreviations: Adv, advantage; ANOVA, analysis of variance; ASD, group diagnosed with autism spectrum disorder; BKB-SIN, Bamford-Kowal-Bench Speech-in-Noise test; Cond, condition; df, degree of freedom; SRT, speech reception threshold.

Note: Data were analyzed with ANOVA or repeated measures ANOVA; post hoc comparisons were conducted with the Tukey-Kramer multiple comparisons test.

${ }^{\mathrm{a} S}$ Statistically significant.

detected for the analyses conducted with a two-factor ANOVA ( - Table 4). As shown in - Table 4, the effect sizes between the ASD and neurotypical groups were medium to large for most statistically significant results. In addition, several medium to large effect sizes were calculated in subtests where no significant differences were detected.

\section{Discussion}

Across all the questionnaires and behavioral test measures, participants with ASD had significantly poorer ratings or auditory-processing performance than age-matched, neurotypical peers with the exception of four of the nine subtests on the TAPS-3 (i.e., word discrimination, phonological segmentation, number memory forward, and auditory comprehension). However, on the phonological segmentation, number memory forward, and auditory comprehension subtests, medium effect sizes were obtained, suggesting a noteworthy difference between groups. The nonsignificant findings may be related to the small sample sizes of each group. It is also important to note that, although 40 to 42 participants completed the majority of the measures, only 28 participants completed the LIFE questionnaire, which may have impacted this group analysis. The missing data was due to noncompliance in all but one of these participants; therefore, we do not believe there would be substantial difference in ratings from responders and nonresponders. Examination of group effect sizes for the tests and subtests with significant results reveal medium, large, or very large effect sizes for both children and adults on the questionnaire ratings as well as most behavioral measures. However, small effect sizes were found for adults on the LiSN-S advantage conditions as well as the phonological blending and word discrimination conditions on the TAPS-3. Obviously, 


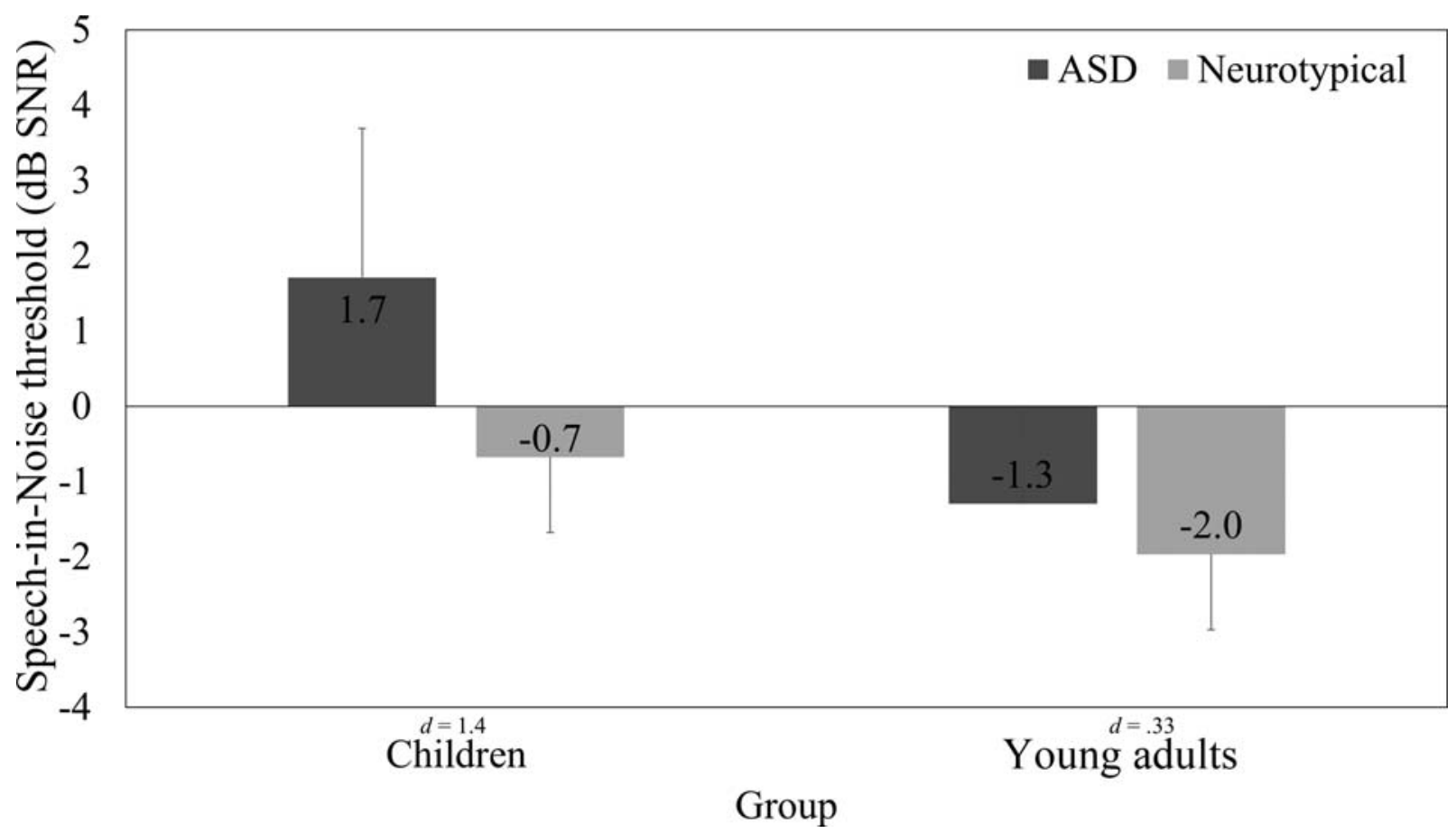

Fig. 2 Average speech-in-noise thresholds on the Bamford-Kowal-Bench Speech-in-Noise (BKB-SIN) test. Cohen's effect sizes between groups are shown above the test and group axis labels. Lower thresholds indicate better performance. Vertical bars represent one standard deviation. ASD, autism spectrum disorder.

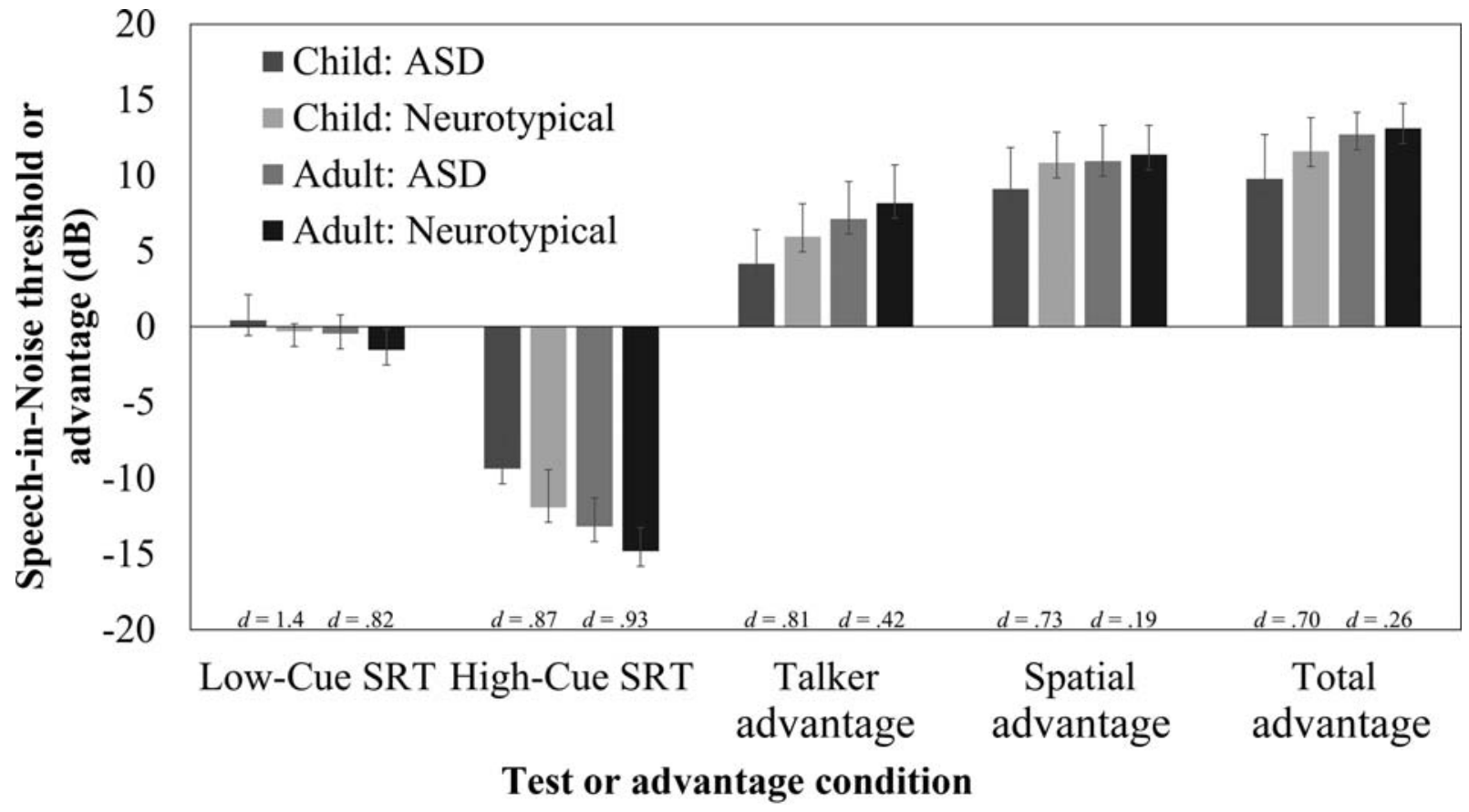

Fig. 3 Average results on the Listening in Spatialized Noise - Sentence Test. Cohen's effect sizes between groups are shown below the corresponding bars. Lower speech reception thresholds (SRTs) indicate better performance while higher advantage scores indicate better use of vocal and spatial cues. Vertical bars represent one standard deviation. ASD, autism spectrum disorder.

performance of all participants with ASD is influenced by the ASD as well as the comorbid disorders commonly associated with ASD. In particular, the majority of the participants with ASD had attention deficits, and several of the children had anxiety and language disorders. Although the participants with ASD were high-functioning, there may also have been slight differences in cognitive ability between groups. Nonetheless, these data reflect the auditory performance of highfunctioning individuals with ASD.

In addition to the group differences, significant age-related differences were detected between the adults and children for the following auditory-processing skills: speech 
Table 3 Summary of nonparametric statistical analyses on Randomized Dichotic Digits test (RDDT) and Dichotic Words test (DWT)

\begin{tabular}{|c|c|c|c|c|c|}
\hline Test & Factors & df & F-statistic & $p$ & Interpretation and post hoc results \\
\hline \multirow{13}{*}{$\begin{array}{l}\text { Randomized Dichotic } \\
\text { Digits test (RDDT) }\end{array}$} & 1-pair: & & & & \\
\hline & -Age & 1,84 & 1.7 & 0.19 & No effect of age \\
\hline & -Group & 1,84 & 16.3 & $<0.001^{a}$ & Neurotypical better than ASD \\
\hline & -Ear & 1,84 & 2.8 & 0.10 & No difference between ears \\
\hline & 2-pair: & & & & \\
\hline & -Age & 1,84 & 1.9 & 0.18 & No effect of age \\
\hline & -Group & 1,84 & 26.2 & $<0.001^{\mathrm{a}}$ & Neurotypical better than ASD \\
\hline & -Ear & 1,84 & 10.4 & $0.01^{\mathrm{a}}$ & Dominant better than non-dominant ear \\
\hline & 3-pair: & & & & \\
\hline & -Age & 1,84 & 0.22 & 0.88 & No effect of age \\
\hline & -Group & 1,84 & 24.4 & $<0.001^{\mathrm{a}}$ & Neurotypical better than ASD \\
\hline & -Ear & 1,84 & 6.6 & $0.01^{\mathrm{a}}$ & Dominant better than non-dominant ear \\
\hline & \multirow[t]{9}{*}{ Ear advantage } & 1-pair: & & & \\
\hline -Age & & 1,42 & 0.11 & 0.74 & No effect of age \\
\hline -Group & & 1,42 & 6.8 & $0.01^{\mathrm{a}}$ & Larger advantage in ASD \\
\hline 2-pair: & & & & & \\
\hline -Age & & 1,42 & 0.41 & 0.53 & No effect of age \\
\hline -Group & & 1,42 & 11.4 & $0.01^{\mathrm{a}}$ & Larger advantage in ASD \\
\hline 3-pair: & & & & & \\
\hline -Age & & 1,42 & 0.01 & 0.91 & No effect of age \\
\hline -Group & & 1,42 & 3.2 & 0.08 & No effect of group \\
\hline \multirow[t]{3}{*}{ Dichotic Words test (DWT) } & -Age & 1,84 & 10.1 & $0.01^{\mathrm{a}}$ & Adults better than children \\
\hline & -Group & 1,84 & 12.7 & $0.01^{a}$ & Neurotypical better than ASD \\
\hline & -Ear & 1,84 & 13.8 & $0.01^{\mathrm{a}}$ & Dominant better than nondominant ear \\
\hline \multirow[t]{2}{*}{ Ear advantage } & -Age & 1,42 & 2.1 & 0.15 & No effect of age \\
\hline & -Group & 1,42 & 4.1 & $0.05^{\mathrm{a}}$ & Larger advantage in ASD \\
\hline
\end{tabular}

Abbreviations: ASD, group diagnosed with autism spectrum disorder; $\mathrm{df}$, degree of freedom.

Note: Data were analyzed with Kruskal-Wallis test; post hoc comparisons were conducted with the Tukey-Kramer multiple comparisons test. Ear refers to dominant versus nondominant scores.

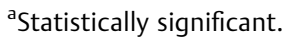

recognition in noise, spatial stream segregation, binaural integration of words, word discrimination, phonological segmentation and blending, number memory reversed, word memory, sentence memory, auditory comprehension, and auditory reasoning. These findings are not surprising given the developmental effects of many auditory-processing skills, as well as the linguistic complexity of many of the subtests. For example, no effects of age were found for dichotic-digit stimuli, which are essentially closed set, when compared with dichotic words. Similarly, no effects of age were detected for the subtests involving digits, but age differences were detected for all other subtests involving phonemes, words, and sentences. As a result, it is important to recognize that, on these particular clinical tests of auditory processing, linguistic complexity is likely to impact performance in children with ASD, as could other possible cognitive factors including attention and memory.
Given the multisensory deficits in individuals with ASD, it is imperative to provide multidisciplinary assessments for this population. For auditory-processing assessments in particular, the team should include an audiologist and a speech-language pathologist who can help to ensure that language levels are considered when interpreting test results. However, as long as children with ASD can reliably complete the testing, the test results can be interpreted and discussed in the context of the child's language functioning. The results of these tests can serve to identify specific auditory-processing skills that may benefit from interventions directed by the audiologist as well as the speechlanguage pathologist such as auditory training. ${ }^{13,28}$ Future research will investigate additional questionnaires and auditory-processing test measures to support a sensitive test battery that may be used clinically and will continue to examine the impact of comorbid conditions and cognitive 


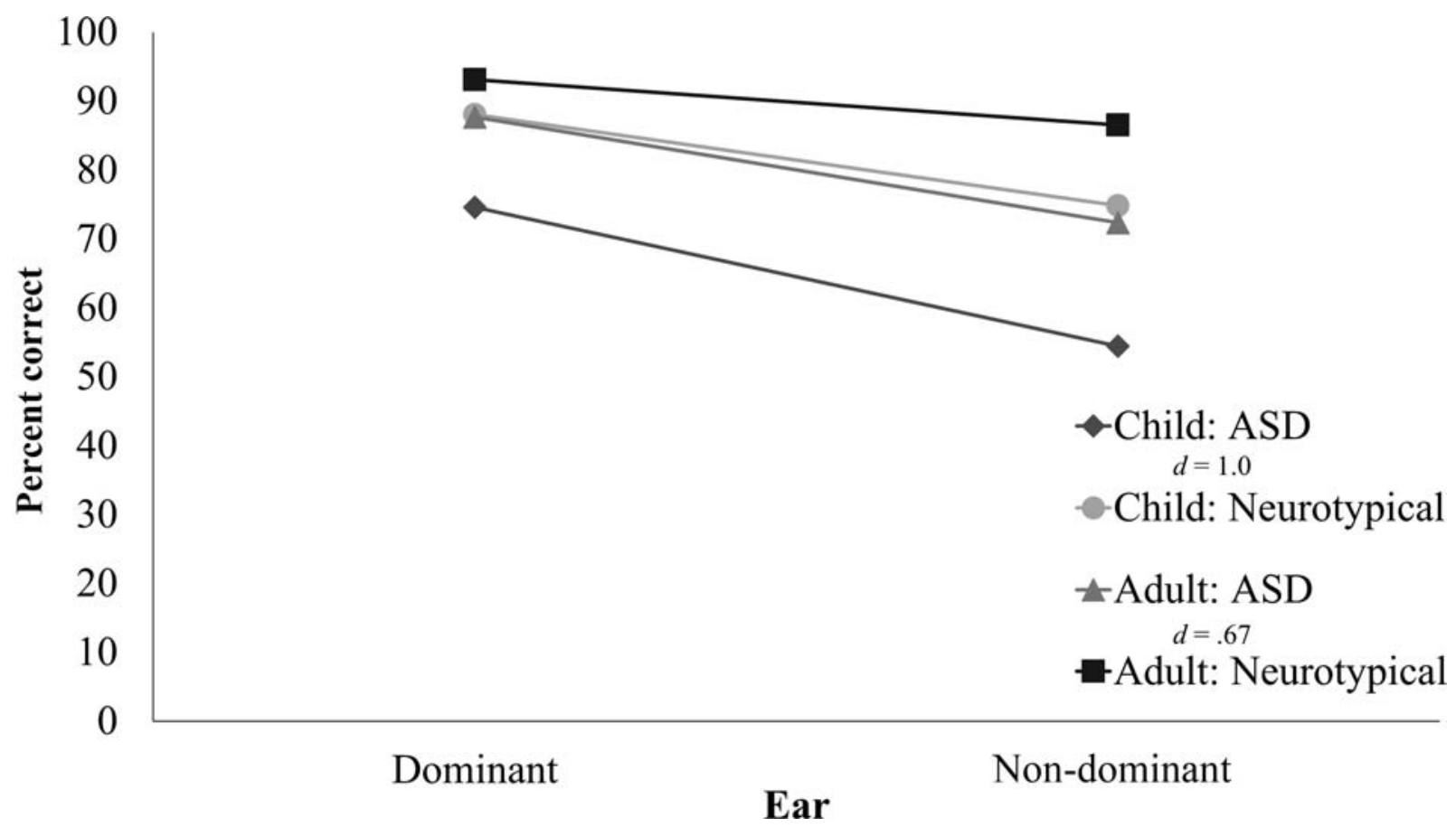

Fig. 4 Average scores on the Dichotic Words test. Effect sizes were collapsed across ears and are shown in the figure legend for children and adults. ASD, autism spectrum disorder.

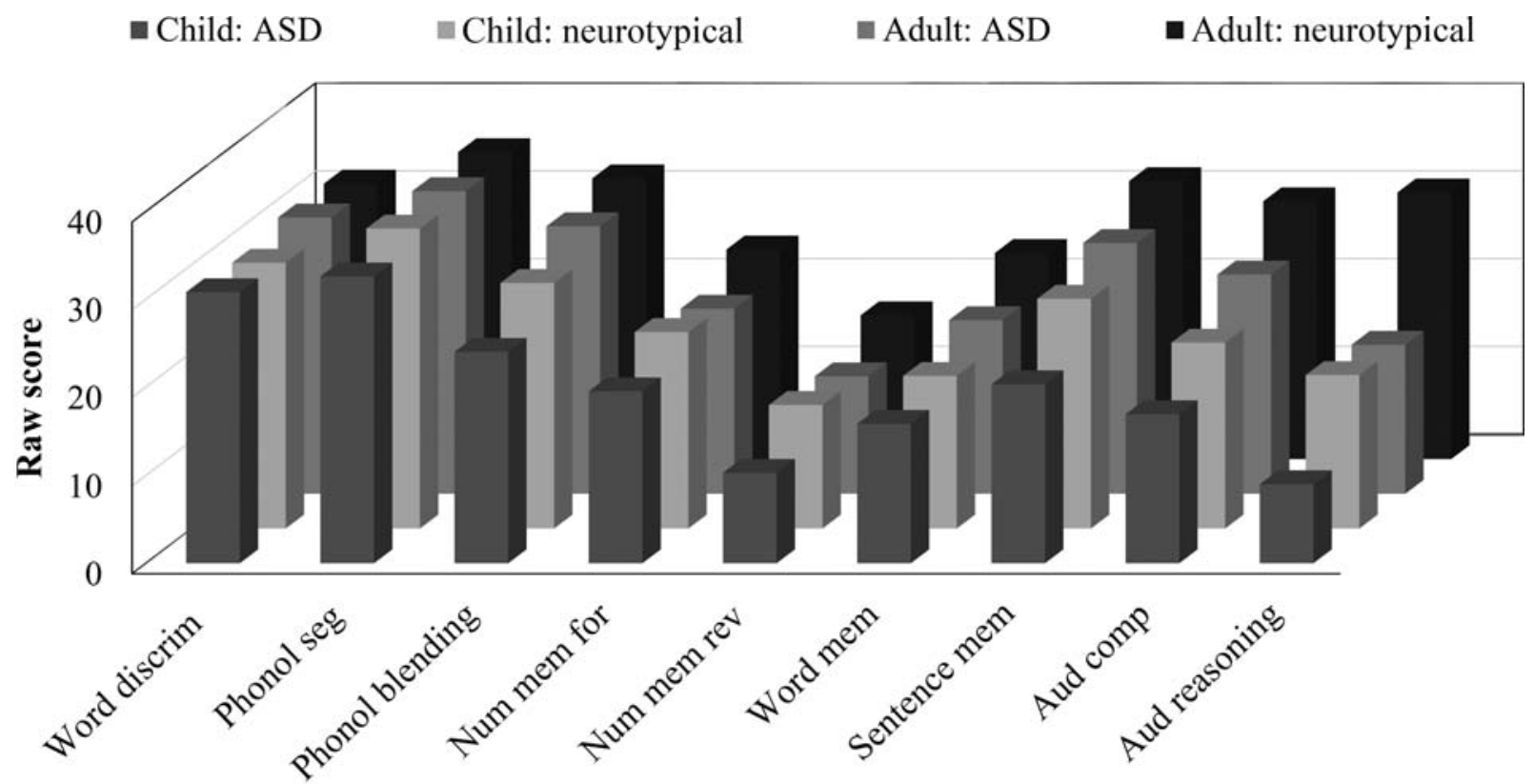

Subtest

Fig. 5 Average raw scores on the Test of Auditory Processing Skills - Third Edition. Aud, auditory; Comp, comprehension; Discrim, discrimination; For, forward; Mem, memory; Num, number; Phonol, phonological; Rev, reversed; Seg, segmentation.

factors, such as attention, memory, and reasoning, on auditory processing.

The clinical implications of this study are noteworthy because the data suggest deficits in almost every area of auditory processing tested in this study. Similar deficits are reported by other investigators. ${ }^{6,12}$ Not only are group differences found on the behavioral test measures, but they are also documented in questionnaires that assess capabilities in real-world listening situations. Our multidisciplinary research team recommends auditory-processing testing for all school-aged children with ASD who can complete the testing reliably. Despite the presence of comorbid conditions, many children are able to complete the testing, thus allowing the team to make specific listening recommendations for home and school environments. Accommodations may include repetition of auditory information, frequent checks for 
Table 4 Summary of statistical analyses on the Test of Audiology Processing Skills (TAPS-3)

\begin{tabular}{|c|c|c|c|c|c|}
\hline Subtest, effects, and factors & df & F-statistic & $p$ & $\begin{array}{l}\text { Interpretation and post } \\
\text { hoc results }\end{array}$ & $\begin{array}{l}\text { Effect sizes between } \\
\text { groups }\end{array}$ \\
\hline \multicolumn{6}{|l|}{ Word discrimination $^{a}$} \\
\hline -Main: Age & 1,40 & 6.2 & $0.02^{c}$ & Adults better than children & \\
\hline -Main: Group & 1,40 & 1.1 & 0.31 & No effect & Child: 0.46; Adult: 0.13 \\
\hline -Interaction: Age/Group & 1,40 & .54 & 0.47 & No interaction & \\
\hline \multicolumn{6}{|l|}{ Phonol segmentation ${ }^{\mathrm{b}}$} \\
\hline -Age & 1,40 & 4.1 & $0.05^{c}$ & Adults better than children & \\
\hline -Group & 1,40 & 2.3 & 0.13 & No effect & Child: 0.34 ; Adult: 0.58 \\
\hline \multicolumn{6}{|l|}{ Phonol blending $^{a}$} \\
\hline -Main: Age & 1,40 & 14.9 & $<0.001^{c}$ & Adults better than children & \\
\hline -Main: Group & 1,40 & 4.0 & $0.05^{c}$ & Neurotypical better than ASD & Child: 0.79; Adult: 0.43 \\
\hline -Interaction: Age/Group & 1,40 & .79 & 0.38 & No interaction & \\
\hline \multicolumn{6}{|l|}{ Num Mem forward ${ }^{\mathrm{a}}$} \\
\hline -Main: Age & 1,40 & .97 & 0.33 & No effect & \\
\hline -Main: Group & 1,40 & 3.4 & 0.07 & No effect & Child: 0.53 ; Adult: 0.63 \\
\hline -Interaction: Age/Group & 1,40 & .00 & 1.0 & No interaction & \\
\hline \multicolumn{6}{|l|}{ Num Mem reversed ${ }^{\mathrm{a}}$} \\
\hline -Main: Age & 1,40 & 3.3 & 0.07 & No effect & \\
\hline -Main: Group & 1,40 & 5.2 & $0.03^{c}$ & Neurotypical better than ASD & Child: 0.72 ; Adult: 0.73 \\
\hline -Interaction: Age/Group & 1,40 & .06 & 0.81 & No interaction & \\
\hline \multicolumn{6}{|l|}{ Word memory ${ }^{\mathrm{a}}$} \\
\hline -Main: Age & 1,40 & 20.6 & $<0.001^{c}$ & Adults better than children & \\
\hline -Main: Group & 1,40 & 5.6 & $0.02^{c}$ & Neurotypical better than ASD & Child: 0.37; Adult: 1.4 \\
\hline -Interaction: Age/Group & 1,40 & 1.0 & 0.32 & No interaction & \\
\hline \multicolumn{6}{|l|}{ Sentence memory ${ }^{\mathrm{a}}$} \\
\hline -Main: Age & 1,40 & 13.0 & $0.01^{c}$ & Adults better than children & \\
\hline -Main: Group & 1,40 & 5.5 & $0.02^{c}$ & Neurotypical better than ASD & Child: 1.0; Adult: 0.50 \\
\hline -Interaction: Age/Group & 1,40 & .51 & 0.48 & No interaction & \\
\hline \multicolumn{6}{|l|}{ Auditory Comp ${ }^{\mathrm{b}}$} \\
\hline - Age & 1,40 & 10.9 & $0.01^{c}$ & Adults better than children & \\
\hline -Group & 1,40 & 2.5 & 0.12 & No effect & Child: 0.15 ; Adult: 0.60 \\
\hline \multicolumn{6}{|l|}{ Auditory reasoning ${ }^{\mathrm{b}}$} \\
\hline -Age & 1,40 & 9.3 & $0.01^{c}$ & Adults better than children & \\
\hline -Group & 1,40 & 10.5 & $0.01^{c}$ & Neurotypical better than ASD & Child: 0.91 ; Adult: 2.0 \\
\hline
\end{tabular}

Abbreviations: ANOVA, analysis of variance; ASD, autism spectrum disorder; Comp, comprehension; df, degree of freedom; Mem, Memory; Num, number; Phonol, phonological.

${ }^{a}$ Data were analyzed with ANOVA.

bData were analyzed using the Kruskal-Wallis test. Post hoc comparisons were conducted with the Tukey-Kramer multiple comparisons test.

'Statistically significant. 
understanding, strategic seating, notetakers, listening breaks, and acoustic modifications (e.g., ceiling tiles). Evidence-based interventions may include the use of bilateral remote-microphone technology, one-on-one auditory training in the presence of background noise, and dichotic listening training to improve binaural integration abilities. $^{11,12,14,15,28-31}$ Continued research will focus on additional evidence-based interventions for the ASD population.

\section{Meeting Presentation}

Portions of this data were presented in a poster presentation at the American Academy of Audiology Conference in 2019, and pre-test data from some of the participants was included in a the Schafer et al study in JAAA, published in May 2019.

\section{Conflict of Interest}

Dr. Schafer reports a grant from the Texas Higher Education Coordinating Board, during the conduct of the study; personal fees from American Academy of Audiology, outside the submitted work. Mrs. Mathews reports grants from Texas Higher Education Coordinating Board, during the conduct of the study. Ms. Canale reports grants from Texas Higher Education Coordinating Board, during the conduct of the study. Ms. Creech reports a receiving funding as a research assistant from a grant from the Texas Higher Education Coordinating Board. Dr. Kaiser reports a receiving funding as a research assistant from a grant from the Texas Higher Education Coordinating Board. The other authors have no conflict of interest to disclose.

\section{References}

1 Baio J, Wiggins L, Christensen DL, et al. Prevalence of autism spectrum disorder among children aged 8 years - Autism and Developmental Disabilities Monitoring Network, 11 Sites, United States, 2014. MMWR Surveill Summ 2018;67(6, No. SS-6);1-23

2 Ashburner J, Ziviani J, Rodger S. Sensory processing and classroom emotional, behavioral, and educational outcomes in children with autism spectrum disorder. Am J Occup Ther 2008;62(05): 564-573

3 Jussila K, Junttila M, Kielinen M, et al. Sensory abnormality and quantitative autism traits in children with and without autism spectrum disorder in an epidemiological population. J Autism Dev Disord 2020;50(01):180-188

4 Leekam SR, Nieto C, Libby SJ, Wing L, Gould J. Describing the sensory abnormalities of children and adults with autism. J Autism Dev Disord 2007;37(05):894-910

5 Tomchek SD, Dunn W. Sensory processing in children with and without autism: a comparative study using the short sensory profile. Am J Occup Ther 2007;61(02):190-200

6 Alcántara JI, Weisblatt EJ, Moore BC, Bolton PF. Speech-in-noise perception in high-functioning individuals with autism or Asperger's syndrome. J Child Psychol Psychiatry 2004;45(06): 1107-1114

7 DePape A-MR, Hall GBC, Tillmann B, Trainor LJ. Auditory processing in high-functioning adolescents with Autism Spectrum Disorder. PLoS One 2012;7(09):e44084

8 Dunlop WA, Enticott PG, Rajan R. Speech discrimination difficulties in high-function autism spectrum disorder are likely independent of auditory hypersensitivity. Front Hum Neurosci 2016; 10(10):401
9 Groen WB, van Orsouw L, Huurne Nt, et al. Intact spectral but abnormal temporal processing of auditory stimuli in autism. J Autism Dev Disord 2009;39(05):742-750

10 Kozou H, Azouz HG, Abdou RM, Shaltout A. Evaluation and remediation of central auditory processing disorders in children with autism spectrum disorders. Int J Pediatr Otorhinolaryngol 2018;104:36-42

11 Rance G, Chisari D, Saunders K, Rault JL. Reducing listeningrelated stress in school-aged children with autism spectrum disorder. J Autism Dev Disord 2017;47(07):2010-2022

12 Rance G, Saunders K, Carew P, Johansson M, Tan J. The use of listening devices to ameliorate auditory deficit in children with autism. J Pediatr 2014;164(02):352-357

13 Schafer EC, Gopal KV, Mathews L, et al. Effects of auditory training and remote microphone technology on the behavioral performance of children and young adults who have autism spectrum disorder. J Am Acad Audiol 2019;30(05):431-443

14 Schafer EC, Mathews L, Mehta S, et al. Personal FM systems for children with autism spectrum disorders (ASD) and/or attentiondeficit hyperactivity disorder (ADHD): an initial investigation. J Commun Disord 2013;46(01):30-52

15 Schafer EC, Wright S, Anderson C, et al. Assistive technology evaluations: remote-microphone technology for children with Autism Spectrum Disorder. J Commun Disord 2016;64:1-17

16 Schelinski S, von Kriegstein K. Brief Report: Speech-in-noise recognition and the relation to vocal pitch perception in adults with autism spectrum disorder and typical development. J Autism Dev Disord 2020;50(01):356-363

17 Duquesnoy AJ. The intelligibility of sentences in quiet and in noise in aged listeners. J Acoust Soc Am 1983;74(04):1136-1144

18 Hammill DD, Pearson NA, Weiderholt JL. Comprehensive Test of Nonverbal Intelligence. 2nd ed. Austin, TX: PRO-ED; 2009

19 Anderson K, Smaldino J, Spangler C. Listening Inventory for Education-Revised (L.I.F.E.-R.). 2011. Available at: https://successforkidswithhearingloss.com/. Accessed January 1, 2015

20 Dunn W. Sensory Profile 2 Manual. San Antonio: Pearson; 2014

21 Brown C, Dunn W. Adolescent/Adult Sensory Profile. San Antonio: Pearson; 2002

22 BKB-SIN. BKB-SIN: Bamford-Kowal-Bench Speech in Noise Test. Elk Grove Village, IL: Etymotic Research; 2005

23 Cameron S, Dillon H. The Listening in Spatialized Noise-Sentences test (LISN-S): comparison to the prototype LISN and results from children with either a suspected (central) auditory processing disorder or a confirmed language disorder. J Am Acad Audiol 2008;19(05):377-391

24 Cameron S, Dillon H. Listening in Spatialized Noise - Sentences Test (Version 2.400) [Computer Software]. Sydney, Australia: National Acoustic Laboratories; 2007

25 Martin NA, Brownell R. Test of Auditory Processing Skills. 3rd ed. Novato: Academy Therapy Publications; 2005

26 Cohen J. The Analysis of Variance. In Statistical Power Analysis for the Behavioral Sciences. 2nd ed. Hilldale, NJ: Lawrence Erlbaum Associates, Publishers; 1988:273-406

27 Lenhard W, Lenhard A. Calculation of Effect Sizes. Dettelbach, GermanyPsychometrica2016

28 Gopal KV, Schafer EC, Mathews L, et al. Effects of auditory training on electrophysiological measures in individuals with autism spectrum disorder. J Am Acad Audiol 2020;31(02):96-104

29 Denman I, Banajee M, Hurley A. Dichotic listening training in children with autism spectrum disorder: a single subject design. Int J Audiol 2015;54(12):991-996

30 Loo JH, Rosen S, Bamiou DE. Auditory training effects on the listening skills of children with auditory processing disorder. Ear Hear 2016;37(01):38-47

31 Moncrieff D, Keith W, Abramson M, Swann A. Evidence of binaural integration benefits following ARIA training for children and adolescents diagnosed with amblyaudia. Int J Audiol 2017;56 (08):580-588 